\title{
EXISTENCE AND UNIQUENESS FOR A QUASISTATIC FRICTIONAL BILATERAL CONTACT PROBLEM IN THERMOVISCOELASTICITY
}

\author{
BY \\ M. ROCHDI (Iremia - University of La Réunion, Saint-Denis, La Réunion, France)
}

AND

M. SHILLOR (Department of Mathematics and Statistics, Oakland University, Rochester, MI)

\begin{abstract}
We prove the existence and uniqueness of the weak solution for a quasistatic thermoviscoelastic problem which describes bilateral frictional contact between a deformable body and a moving rigid foundation. The model consists of the heat equation for the temperature, the elliptic viscoelasticity system for the displacements, the SJK-Coulomb law of friction and frictional heat generation condition. The proof is accomplished in two steps. First, the existence of solutions for a regularized problem is established and a priori estimates obtained. Then the limit function, which is the weak solution of the original problem, is shown to be the unique fixed point of the solution operator when the friction coefficient is small.
\end{abstract}

1. Introduction. This work deals with a model for the quasistatic process of bilateral frictional contact between a viscoelastic body and a rigid moving foundation. The model consists of the heat equation for the temperature and the elliptic viscoelasticity system for the displacements, together with friction and frictional heat generation conditions on the contact surface. We establish the existence and uniqueness of the weak solution for the problem when the coefficient of friction is sufficiently small. The proof is based on the study of a sequence of auxiliary problems, passage to the limit when the regularization parameter vanishes, and an application of a fixed point argument.

The model describes the evolution of the thermomechanical state of a part or component that is in frictional contact with a harder object. The assumption that the acting forces, tractions and possible heat sources change gradually in time allows us to neglect the inertial terms in the equations of motion and use the quasistatic approximation. In particular, we neglect any viscoelastic waves in the body, which is the case in a system such as a pump or a low rpm motor, where slow periodic forces act on the contacting

Received June 30, 1998.

2000 Mathematics Subject Classification. Primary 70E18; Secondary 35R45, 35J85, 35K85.

Key words and phrases. Quasistatic frictional contact, viscoelastic, bilateral contact, frictional heat generation, fixed point, SJK-Coulomb law of friction. 
elements. Often in such systems, the contacting elements are partially lubricated and, therefore, the friction coefficient is small.

There exists a large body of research on frictional contact in the engineering literature. Recent results from a number of different points of view can be found in [23] and references therein. General dynamic thermoelastic models, which were derived from thermodynamical principles, can be found in $[13,25]$. The mathematical literature on dynamic or quasistatic frictional contact models that include thermal effects is very recent, although in applications frictional contact is very often accompanied by considerable heat generation. Indeed, when car brakes are applied, the frictional heat generation can easily exceed 100 HP. Dynamic thermoviscoelastic frictional contact problems can be found in $[4,5,10,20]$. In [5] the wear of the contacting surface was included. A one-dimensional thermoviscoelastic problem for a beam was investigated in [15] where the existence of the weak solution was established and numerical simulations of the solutions conducted. A quasistatic thermoviscoelastic problem for a beam can be found in $[12,11]$ where the wear of the contacting end is included. A frictionless one-dimensional thermoelastic contact problem for a rod was thoroughly analyzed in [3].

Recent existence and uniqueness results for quasistatic contact problems can be found in $[2,7,18,21,22,24]$. However, this paper is the first to investigate the general bilateral quasistatic contact problem in three dimensions in which the thermal effects of friction are taken into account.

The behavior of the bulk material is assumed, for the sake of simplicity, to be linear. The nonlinear effects on which we focus occur on the part of the boundary that is in contact with the rigid foundation. We employ the Kelvin-Voigt viscoelastic law with thermal effects included. We model friction by the SJK-version of Coulomb's law, [25], and assume that the contact is maintained throughout the process, which is the case in many engineering applications. The frictional heat generation enters as a boundary condition for the temperature.

In Sec. 2 we present the physical setting and formulate the model as a coupled system of parabolic-elliptic partial differential equations, together with initial and boundary conditions. Because of the friction condition there is a regularity ceiling for the solutions and, in general, these problems do not admit classical solutions. Therefore, we introduce the weak formulation in the form of a variational inequality and then rewrite it in an abstract operator form. Then we list the assumptions imposed on the data and state our main existence and uniqueness result in Theorem 2.2. It guarantees that when the coefficient of friction is small, the problem has a unique weak solution. Estimating the bound on the friction coefficient remains an important unsolved question; however, partially lubricated surfaces have small friction coefficients. Finally, we present an auxiliary problem in which the contact stress is assumed to be known. The existence of the unique solution of the regularized version of the auxiliary problem is established in Sec. 3 where we use an abstract existence result for degenerate evolution equations due to [14]. We note in passing that a considerable generalization of this theorem can be found in [16]. Then we obtain the necessary a priori estimates on the solutions. We pass to the regularization limit in Sec. 4 and, thus, prove the existence of the unique solution 
to the auxiliary problem. Finally, in Sec. 5 we use a fixed point argument and establish Theorem 2.2.

There remain many open questions, in addition to estimating the size of the friction coefficient. Does the solution converge to the steady solution when the forces, tractions and heat sources converge to time-independent quantities? If so, what is the rate of convergence? Moreover, the wear of the contacting surfaces needs to be taken into account. Finally, the auxiliary problem and the fixed point argument may be a basis for a convergent numerical algorithm, but will it be sufficiently effective in practice?

2. The model, weak formulation and results. The physical setting consists of a viscoelastic body which, over a part of its surface, is in frictional contact with a rigid moving foundation. The body (in its reference configuration) is represented by $\Omega$, a region in $\mathbb{R}^{m}(m=2,3)$, whose boundary $\partial \Omega=\Gamma$, which is assumed to be Lipschitz continuous, is divided into three disjoint parts. On the first part, denoted by $\Gamma_{D}$, the body is clamped; known tractions act on the second part $\Gamma_{N}$; the body is in frictional contact with a rigid obstacle on the third part, $\Gamma_{C}$. The reference configuration is assumed to be stress free and isothermal; its temperature is conveniently set as zero, which also serves as the reference temperature. The rigid foundation moves with tangential velocity $\phi$ and this motion is accompanied by frictional heat generation - which is considerable in many applications - on the part of the contact surface where relative slip takes place.

We assume that contact is maintained between the body and the foundation. This is the case in many engineering systems where there is no loss of contact, such as between the piston rings and the engine block in a car. An example from everyday life is the frictional contact of the wheels with the rail when a train is braking. From the mathematical point of view the approach below does not allow us to consider the case when separation occurs between the body and the foundation.

We assume that the applied forces, tractions and the heat source vary slowly with time and consequently neglect the accelerations in the system. So we employ the quasistatic approximation for the process. The unknowns in the model are the displacement vector $\mathbf{u}=\left(u_{1}(x, t), \ldots, u_{m}(x, t)\right)$, the temperature $\theta=\theta(x, t)$, and the stress tensor $\sigma=$ $\sigma_{i j}(x, t)$, at location $x$ and time $t$.

The model for the process of quasistatic thermoviscoelastic bilateral contact with friction is as follows:

Find $\{\mathbf{u}, \sigma, \theta\}$ such that

$$
\begin{aligned}
\sigma_{i j}=a_{i j k l} u_{k, l}+b_{i j k l} u_{k, l}^{\prime}-c_{i j} \theta & \text { in } \Omega_{T}, \\
\sigma_{i j, j}+f_{A i}=0 & \text { in } \Omega_{T}, \\
\theta^{\prime}-\left(k_{i j} \theta_{, j}\right)_{, i}=-c_{i j} u_{i, j}^{\prime}+q & \text { in } \Omega_{T}, \\
\mathbf{u}=0 & \text { on } \Gamma_{D} \times(0, T), \\
\sigma \mathbf{n}=\mathbf{f}_{N} & \text { on } \Gamma_{N} \times(0, T), \\
\theta=\theta_{b} & \text { on }\left(\Gamma_{D} \cup \Gamma_{N}\right) \times(0, T),
\end{aligned}
$$




$$
\begin{array}{cl}
u_{n}=0 & \text { on } \Gamma_{C} \times(0, T), \\
\left|\sigma_{\tau}\right| \leq \mu\left|R \sigma_{n}\right|\left(1-\delta\left|R \sigma_{n}\right|\right)_{+} & \text {on } \Gamma_{C} \times(0, T), \\
\left|\sigma_{\tau}\right|<\mu\left|R \sigma_{n}\right|\left(1-\delta\left|R \sigma_{n}\right|\right)_{+} & \Rightarrow \mathbf{u}_{\tau}^{\prime}=\phi, \\
\left|\sigma_{\tau}\right|=\mu\left|R \sigma_{n}\right|\left(1-\delta\left|R \sigma_{n}\right|\right)_{+} & \Rightarrow \mathbf{u}_{\tau}^{\prime}=\phi-\lambda \sigma_{\tau}, \lambda \geq 0, \\
k_{i j} \theta_{, i} n_{j}=\mu\left|R \sigma_{n}\right|\left(1-\delta\left|R \sigma_{n}\right|\right)_{+} s_{C}\left(\cdot,\left|\mathbf{u}_{\tau}^{\prime}-\phi\right|\right) & \\
-k_{e}\left(\theta-\theta_{R}\right) & \text { on } \Gamma_{C} \times(0, T), \\
\mathbf{u}(\cdot, 0)=\mathbf{u}_{0}, \theta(\cdot, 0)=\theta_{0} & \text { in } \Omega .
\end{array}
$$

Here, $\Omega_{T}=\Omega \times(0, T), i, j=1, \ldots, m$, the repeated index convention is employed, the prime represents time derivative, the portion of a subscript prior to a comma indicates a component and the portion after the comma refers to partial derivatives. All the variables are scaled and form dimensionless quantities.

The thermoviscoelastic constitutive relation is given in $(2.1)$ where $a=\left(a_{i j k l}\right)$ and $b=\left(b_{i j k l}\right)$ are the tensors of elastic and viscosity coefficients, respectively. Since we consider the quasistatic approximation for the process, (2.2) represents the equilibrium equations, and $\mathbf{f}_{A}=\left(f_{A 1}(x, t), \ldots, f_{A m}(x, t)\right)$ denotes the density of applied body forces acting in $\Omega$. For the sake of simplicity, the material density is assumed constant and set equal to one. (2.3) is the energy equation where $c_{i j}$ and $k_{i j}$ are the components of the thermal expansion and thermal conductivity tensors, respectively, and $q$ is the density of volume heat sources.

To describe the boundary conditions we introduced the unit outward normal $\mathbf{n}=$ $\left(n_{1}, \ldots, n_{m}\right)$ to $\Gamma$, and since $\Gamma$ is assumed Lipschitz, $\mathbf{n}$ exists at almost every point there. We denote by $\sigma_{n}=\sigma_{i j} n_{i} n_{j}$ and $u_{n}=u_{i} n_{i}$ the normal components of $\sigma$ and $\mathbf{u}$ on $\Gamma$, respectively, and let

$$
\sigma_{\tau}=\sigma \mathbf{n}-\sigma_{n} \mathbf{n}, \quad \mathbf{u}_{\tau}=\mathbf{u}-u_{n} \mathbf{n}
$$

be the tangential parts (see, e.g., [17] or [9]).

In $(2.5)$ and $(2.6), \mathbf{f}_{N}=\left(f_{N 1}(x, t), \ldots, f_{N m}(x, t)\right)$ denotes the tractions applied to $\Gamma_{N}$, and $\theta_{b}$ is the known and scaled temperature of the part of the boundary $\Gamma_{D} \cup \Gamma_{N}$. Condition (2.7) means that there is no loss of contact.

We turn to the conditions on the contact surface. In (2.8) we employ the SJKgeneralization of Coulomb's law of dry friction ([25]). $\mu$ is the friction coefficient, $\delta$ is a small positive coefficient related to the wear and hardness of the surface, $\mathbf{u}_{\tau}^{\prime}$ is the tangential velocity of the body, $(\cdot)_{+}=\max (\cdot, 0)$ and $R$ represents a normal regularization operator, a linear and continuous operator $R: H^{-\frac{1}{2}}(\Gamma) \rightarrow L^{2}(\Gamma)$ (see, e.g., [8]). We use it in (2.8) and (2.9) to regularize the trace of the stress tensor on $\Gamma$. Condition (2.8) may be interpreted physically in the following way. The boundary sticks to the foundation and moves with it when the applied tangential stress is less than the limiting value. The part of $\Gamma_{C}$ where this takes place is called the stick zone. The part where the tangential stress reaches its limiting value and does not move in tandem with the foundation is called the slip zone. $\lambda$ is an unknown multiplier that indicates the relative direction of the slip. 
The modification to Coulomb's law of friction, which was derived in [25] from thermodynamical considerations, consists of the factor $(1-\delta|\cdot|)_{+}$. It represents the fact that when the contact stress is large, there is a decrease in frictional resistance because of surface wear. This modified condition agrees with Coulomb's law for $\left|\sigma_{n}\right|$ not too large since $\delta$ is very small in applications. On the other hand, when the contact tractions are very large it is very likely that the surface will be damaged, and Coulomb's condition, actually the whole model, needs to be redone. Thus, from the point of view of applications, the modified condition seems more natural. From the mathematical point of view, the fact that the tangential stress is bounded is essential to obtaining the necessary estimates below.

Next, the introduction of the regularization operator $R$ is necessary since the contact stress is not sufficiently regular to make the condition of friction meaningful. Indeed, $\sigma$ on $\Gamma_{C}$ lies in $H^{-\frac{1}{2}}(\Gamma)$, which means that it is only a distribution, too irregular to use below. An explanation of the mathematical difficulties and a justification for using $R$ can be found in [8]. The operator $R$ may be chosen to have a small support, in which case it will average the contact stress over the surface asperities in a small region on $\Gamma_{C}$. We do not know how it affects the friction bound since we cannot obtain comparable results without it.

We now describe the temperature boundary condition (2.9) on $\Gamma_{C}$. The power generated by the frictional contact forces is proportional to $\left|\sigma_{\tau}\right|$ and $\left|\mathbf{u}_{\tau}^{\prime}-\phi\right|$; so, for the sake of generality, we used the function $s_{c}(\cdot, r)$, which is prescribed and generalizes $|\cdot|$, and it is given by $\mu\left|R \sigma_{n}\right|\left(1-\delta\left|R \sigma_{n}\right|\right)_{+} s_{c}\left(\cdot,\left|\mathbf{u}_{\tau}^{\prime}-\phi\right|\right) . \theta_{R}$ is the foundation's temperature and $k_{e}$ is the coefficient of heat exchange between it and the body. Finally, (2.10) represents the initial conditions.

It is well known that, in general, there are no classical solutions to the problem because of the regularity ceiling related to the possible stick-slip motion. Therefore, we consider a weak or variational formulation for the problem. To this end we introduce the following classical Hilbert spaces:

$$
\begin{gathered}
H=L^{2}(\Omega), \quad H^{m}=L^{2}(\Omega)^{m}, \\
E=\left\{w \in H^{1}(\Omega)^{m}: w=0 \text { on } \Gamma_{D}\right\}, \\
V=\left\{\eta \in H^{1}(\Omega): \eta=0 \text { on } \Gamma_{D} \cup \Gamma_{N}\right\}, \\
W=\left\{\tau=\left(\tau_{i j}\right): \tau_{i j}=\tau_{j i} \in L^{2}(\Omega), \tau_{i j, j} \in L^{2}(\Omega)\right\}, \\
\mathbb{H}=L^{2}(0, T ; H), \quad \mathbb{E}=L^{2}(0, T ; E), \quad \mathbb{V}=L^{2}(0, T ; V), \quad \mathbb{W}=L^{2}(0, T ; W) .
\end{gathered}
$$

Below, we use $\|\cdot\|_{E},\|\cdot\|_{V}$, and $\|\cdot\|_{W}$ to denote the norms of $E, V$, and $W$, respectively. Similarly, we use $|\cdot|_{H}$ and $|\cdot|_{H^{m}}$ to denote the norms of $H$ and $H^{m}$, respectively, and $\langle\cdot, \cdot\rangle$ denotes the duality pairing between $E^{\prime}$ and $E$ or $V^{\prime}$ and $V$, as the meaning is evident from the context. For standard notation we refer the reader to $[1,19,17]$. 
We now describe the assumptions on the data. The coefficients of elasticity, viscosity, thermal expansion, and thermal conductivity satisfy:

$$
\begin{aligned}
& a_{i j k l} \in L^{\infty}(\Omega), \quad b_{i j k l} \in L^{\infty}(\Omega), \quad c_{i j} \in L^{\infty}(\Omega), \quad k_{i j} \in L^{\infty}(\Omega) ; \\
& a_{i j k l}=a_{j i k l}, \quad a_{i j k l}=a_{k l i j}, \quad a_{i j k l}=a_{i j l k}, \\
& a_{i j k l} \chi_{k l} \chi_{i j} \geq \alpha_{1} \chi_{i j} \chi_{i j} \quad \text { for all symmetric tensors } \chi=\left(\chi_{i j}\right) ; \\
& b_{i j k l}=b_{j i k l}, \quad b_{i j k l}=b_{k l i j}, \quad b_{i j k l}=b_{i j l k}, \\
& b_{i j k l} \chi_{k l} \chi_{i j} \geq \alpha_{2} \chi_{i j} \chi_{i j} \quad \text { for all symmetric tensors } \chi=\left(\chi_{i j}\right) ; \\
& c_{i j}=c_{j i} ; \\
& k_{i j}=k_{j i}, \quad k_{i j} z_{j} z_{i} \geq \alpha_{3} z_{i} z_{i} \quad \text { for all vectors } z=\left(z_{i}\right) .
\end{aligned}
$$

Here, $\alpha_{1}, \alpha_{2}$, and $\alpha_{3}$ are positive constants.

The body forces and the volume heat sources satisfy

$$
\mathbf{f}_{a} \in L^{2}\left(0, T ; H^{m}\right), \quad q \in \mathbb{V}^{\prime} .
$$

The friction coefficient and the velocity of the foundation satisfy

$$
\begin{aligned}
& \mu \in L^{\infty}\left(\Gamma_{C}\right), \quad \mu \geq 0, \quad \text { a.e. on } \Gamma_{C}, \\
& \phi=\phi(t) \in C\left([0, T] ; \mathbb{R}^{m-1}\right) .
\end{aligned}
$$

The function $s_{c}$ satisfies

$$
\begin{aligned}
& s_{c}: \Gamma_{C} \times \mathbb{R} \rightarrow \mathbb{R}_{+} \text {is Borel measurable, } \\
& s_{c}(\cdot, r) \leq \alpha_{4} \quad \text { for all } r \in \mathbb{R}, \quad \text { a.e. on } \Gamma_{C}, \\
& \left|s_{c}\left(\cdot, r_{1}\right)-s_{c}\left(\cdot, r_{2}\right)\right| \leq \alpha_{5}\left|r_{1}-r_{2}\right| \text { for all } r_{1}, r_{2} \in \mathbb{R}, \quad \text { a.e. on } \Gamma_{C},
\end{aligned}
$$

where $\alpha_{4}$ and $\alpha_{5}$ are positive constants.

The assumptions on the boundary and initial data are

$$
\begin{aligned}
& \mathbf{f}_{N} \in L^{2}\left(0, T ; L^{2}\left(\Gamma_{N}\right)^{m}\right) ; \\
& \text { there exists } \Theta \in H^{1}\left(0, T ; H^{1}(\Omega)\right) \text { such that } \Theta=\theta_{b} \text { on } \Gamma_{D} \cup \Gamma_{N} ; \\
& \theta_{R} \in L^{2}\left(0, T ; L^{2}\left(\Gamma_{C}\right)\right) \\
& \mathbf{u}_{0} \in E, \quad \theta_{0} \in H .
\end{aligned}
$$

For technical reasons, it is convenient to shift the temperature function so that it is zero on $\Gamma_{D} \cup \Gamma_{N}$. To that end, we introduce $\xi=\theta-\Theta$ and $\xi_{0}=\theta_{0}-\Theta(\cdot, 0)$. To simplify the notation, we will not indicate explicitly the dependence on $t$.

We can now present the weak formulation of the problem.

Definition 2.1. A triplet $\{\mathbf{u}, \sigma, \theta\}$ is said to be a weak solution to (2.1)-(2.10) provided that it satisfies

$$
\begin{aligned}
& \mathbf{u} \in \mathbb{E}, \quad \mathbf{u}^{\prime} \in \mathbb{E}, \quad \mathbf{u}(\cdot, 0)=\mathbf{u}_{0}, \\
& \xi \in \mathbb{V}, \quad \xi^{\prime} \in \mathbb{V}^{\prime}, \quad \boldsymbol{\xi}(\cdot, 0)=\boldsymbol{\xi}_{0}, \\
& \sigma \in \mathbb{W}, \quad \sigma_{i j}=a_{i j k l} u_{k, l}+b_{i j k l} u_{k, l}^{\prime}-c_{i j}(\xi-\Theta),
\end{aligned}
$$


and for all $w \in \mathbb{E}$,

$$
\begin{gathered}
\int_{\Omega} a_{i j k l} u_{k, l}\left(w_{i, j}-u_{i, j}^{\prime}\right) d x+\int_{\Omega} b_{i j k l} u_{k, l}^{\prime}\left(w_{i, j}-u_{i, j}^{\prime}\right) d x-\int_{\Omega} c_{i j} \xi\left(w_{i, j}-u_{i, j}^{\prime}\right) d x \\
+\int_{\Gamma_{C}} \mu\left|R \sigma_{n}\right|\left(1-\delta\left|R \sigma_{n}\right|\right)_{+}\left(\left|w_{\tau}-\phi\right|-\left|\mathbf{u}_{\tau}^{\prime}-\phi\right|\right) d \Gamma \\
\geq \int_{\Omega} f_{A i}\left(w_{i}-u_{i}^{\prime}\right) d x+\int_{\Omega} c_{i j} \Theta\left(w_{i, j}-u_{i, j}^{\prime}\right) d x+\int_{\Gamma_{N}} f_{N i}\left(w_{i}-u_{i}^{\prime}\right) d \Gamma,
\end{gathered}
$$

and, for all $\eta \in \mathbb{V}$,

$$
\begin{aligned}
\left\langle\xi^{\prime}, \eta\right\rangle+\int_{\Omega} k_{i j} \xi_{, i} \eta_{, j} d x & +\int_{\Omega} c_{i j} u_{i, j}^{\prime} \eta d x+\int_{\Gamma_{C}} k_{e} \xi \eta d x \\
& -\int_{\Gamma_{C}} \mu\left|R \sigma_{n}\right|\left(1-\delta\left|R \sigma_{n}\right|\right)_{+} s_{c}\left(x,\left|\mathbf{u}_{\tau}^{\prime}-\phi\right|\right) \eta d \Gamma \\
= & \langle q, \eta\rangle-\int_{\Omega} \Theta^{\prime} \eta d x-\int_{\Gamma_{C}} k_{e}\left(\Theta-\theta_{R}\right) \eta d \Gamma-\int_{\Omega} k_{i j} \Theta_{, i} \eta_{, j} d x .
\end{aligned}
$$

We write the weak formulation in an abstract form, and to that end we define the following operators:

$$
\begin{array}{r}
A, B: E \rightarrow E^{\prime}, \\
C_{1}: E \rightarrow V^{\prime}, \\
C_{2}: V \rightarrow E^{\prime}, \\
K_{1}, K_{2}: V \rightarrow V^{\prime}, \\
S: W \times E \rightarrow V^{\prime},
\end{array}
$$

by

$$
\begin{aligned}
\langle A u, w\rangle & =\int_{\Omega} a_{i j k l} u_{k, l} w_{i, j} d x, \\
\langle B v, w\rangle & =\int_{\Omega} b_{i j k l} v_{k, l} w_{i, j} d x \\
\left\langle C_{1} v, \eta\right\rangle & =\int_{\Omega} c_{i j} v_{i, j} \eta d x \\
\left\langle C_{2} \xi, w\right\rangle & =-\int_{\Omega} c_{i j} \xi w_{i, j} d x, \\
\left\langle K_{1} \xi, \eta\right\rangle & =\int_{\Gamma_{C}} k_{e} \xi \eta d \Gamma, \\
\left\langle K_{2} \xi, \eta\right\rangle & =\int_{\Omega} k_{i j} \xi_{, i} \eta, j d x, \\
\langle S(\sigma, v), \eta\rangle & =-\int_{\Gamma_{C}} \mu\left|R \sigma_{n}\right|\left(1-\delta\left|R \sigma_{n}\right|\right)_{+} s_{c}\left(x,\left|v_{\tau}-\phi\right|\right) \eta d \Gamma .
\end{aligned}
$$

We note that each of these operators extends, in a natural way, to an operator defined on the corresponding space of square-integrable vector-valued functions on $(0, T)$. For example, $A$ extends to an operator from $\mathbb{E}$ to $\mathbb{E}^{\prime}$ by setting $(A u)(t)=A(u(t))$. With a 
slight abuse of notation, we will use below the same symbol to denote both the original operator and its extension, since the meaning will be clear from the context. We can now formulate Problem (2.16)-(2.20) abstractly as follows:

Problem P: Find $\{\mathbf{u}, \sigma, \xi\}$ satisfying (2.16)-(2.18) and such that

$$
\begin{gathered}
\xi^{\prime}+K_{1} \xi+K_{2} \xi+C_{1} \mathbf{u}^{\prime}+S\left(\sigma, \mathbf{u}^{\prime}\right)=Q \text { in } \mathbb{V}^{\prime} \\
B \mathbf{u}^{\prime}+A \mathbf{u}+C_{2} \xi+\partial_{2} j\left(\sigma, \mathbf{u}^{\prime}\right) \ni f \text { in } \mathbb{E}^{\prime}
\end{gathered}
$$

Here $f \in \mathbb{E}^{\prime}$ and $Q \in \mathbb{V}^{\prime}$ are given by

$$
\begin{array}{r}
\langle f, w\rangle=\int_{0}^{T} \int_{\Omega} f_{A i} w_{i} d x d t+\int_{0}^{T} \int_{\Omega} c_{i j} \Theta w_{i, j} d x d t+\int_{0}^{T} \int_{\Gamma_{N}} f_{N i} w_{i} d \Gamma d t \\
\langle Q, \eta\rangle=\int_{0}^{T}\langle q, \eta\rangle d t-\int_{0}^{T} \int_{\Omega} \Theta^{\prime} \eta d x d t-\int_{0}^{T} \int_{\Gamma_{C}} k_{e}\left(\Theta-\theta_{R}\right) \eta d \Gamma d t \\
-\int_{0}^{T} \int_{\Omega} k_{i j} \Theta_{, i} \eta_{, j} d x d t
\end{array}
$$

respectively, and $\partial_{2} j(\sigma, v)$ denotes the partial subdifferential with respect to $v$ of

$$
j(\sigma, v)=\int_{0}^{T} \int_{\Gamma_{C}} \mu\left|R \sigma_{n}\right|\left(1-\delta\left|R \sigma_{n}\right|\right)_{+}\left|v_{\tau}-\phi\right| d \Gamma d t .
$$

Our main existence and uniqueness result is the following.

Theorem 2.2. Assume that (2.11)-(2.15) hold. Then Problem $P$ has a unique solution when $|\mu|_{L^{\infty}\left(\Gamma_{C}\right)}$ is sufficiently small.

We conclude that problem $(2.1)-(2.10)$ has a unique weak solution when $|\mu|_{L^{\infty}\left(\Gamma_{C}\right)}$ is sufficiently small. Estimating the allowed size of the friction coefficient is an open and very interesting problem.

The proof of Theorem 2.2 is accomplished in several steps. The first consists of studying a problem of the form (2.16)-(2.18), (2.28), and (2.29) when the stress $\sigma$ on the contact boundary is assumed to be known. The existence of a solution for this intermediate problem is obtained as a limit of solutions for a sequence of approximate problems that are presented in the next section.

In the last step of the proof of the theorem we use a fixed point argument and obtain a solution of Problem $P$.

In the first step, for each $g \in \mathbb{W}$ we consider the following abstract problem:

Problem $P_{g}$ : Find $\left\{\mathbf{u}_{g}, \sigma_{g}, \xi_{g}\right\}$ such that

$$
\begin{aligned}
& \mathbf{u}_{g} \in \mathbb{E}, \quad \mathbf{u}_{g}^{\prime} \in \mathbb{E}, \quad \mathbf{u}_{g}(\cdot, 0)=\mathbf{u}_{0}, \\
& \xi_{g} \in \mathbb{V}, \quad \xi_{g}^{\prime} \in \mathbb{V}^{\prime}, \quad \xi_{g}(\cdot, 0)=\xi_{0}, \\
& \sigma_{g} \in \mathbb{W}, \quad\left\{\sigma_{g}\right\}_{i j}=a_{i j k l}\left\{\mathbf{u}_{g}\right\}_{k, l}+b_{i j k l}\left\{\mathbf{u}_{g}^{\prime}\right\}_{k, l}-c_{i j}\left(\xi_{g}-\Theta\right), \\
& \xi_{g}^{\prime}+K_{1} \xi_{g}+K_{2} \xi_{g}+C_{1} \mathbf{u}_{g}^{\prime}+S\left(g, \mathbf{u}_{g}^{\prime}\right)=Q \quad \text { in } \mathbb{V}^{\prime}, \\
& B \mathbf{u}_{g}^{\prime}+A \mathbf{u}_{g}+C_{2} \xi_{g}+\partial_{2} j\left(g, \mathbf{u}_{g}^{\prime}\right) \ni f \quad \text { in } \mathbb{E}^{\prime} .
\end{aligned}
$$

We have the following result.

Theorem 2.3. Assume that (2.11)-(2.15) hold. Then Problem $P_{g}$ has a unique solution. 
The solution of Problem $P_{g}$ will be obtained in the next section.

3. Approximate problems. In this section we consider a sequence of regularized approximations to Problem $P_{g}$. The solutions of these problems are obtained by using a version of an abstract existence theorem for degenerate first-order evolution equations obtained by Kuttler in [14] (see also [16] for a generalization of the theorem) which we now recall.

Let $F$ and $G$ be reflexive Banach spaces such that $F \subseteq G,\|\cdot\|_{F} \geq\|\cdot\|_{G}$ and $F$ is dense in $G$; thus, we may write $F \subseteq G \equiv G^{\prime} \subseteq F^{\prime}$. Suppose that $\mathcal{B}$ is a linear, bounded, positive and symmetric operator from $G$ to $G^{\prime}$. Let $\mathbb{F}=L^{2}(a, b ; F), \mathbb{G}=L^{2}(a, b ; G)$, and define $\mathbb{X}=\left\{w \in \mathbb{F}:(\mathcal{B} w)^{\prime} \in \mathbb{F}^{\prime}\right\}$ with the graph norm $\|w\|_{\mathbb{X}}=\|w\|_{\mathbb{F}}+\left\|(\mathcal{B} w)^{\prime}\right\|_{\mathbb{F}^{\prime}}$. Here, the differentiation is taken in the sense of $F^{\prime}$-valued distributions. It is easy to see that $\mathbb{X}$ is a reflexive Banach space. Let $\mathcal{A}(t, \cdot)$ be an operator from $F$ to $F^{\prime}$. We also denote by $\mathcal{A}$ its natural extension from $\mathbb{F}$ to $\mathbb{F}^{\prime}$ given by $\mathcal{A} w(t)=\mathcal{A}(t, w(t))$. Assume that

$$
\mathcal{A}: \mathbb{X} \rightarrow \mathbb{X}^{\prime} \text { is pseudomonotone }
$$

(see, e.g., $[6])$,

$$
\mathcal{A}: \mathbb{F} \rightarrow \mathbb{F}^{\prime} \text { is bounded, }
$$

and, for some $\lambda \in \mathbb{R}$,

$$
\lim _{\|w\|_{\mathbb{F}} \rightarrow \infty} \frac{\lambda\langle\mathcal{B} w, w\rangle_{G^{\prime} \times G}+\langle\mathcal{A} w, w\rangle_{\mathbb{F}^{\prime} \times \mathbb{F}}}{\|w\|_{\mathbb{F}}}=\infty .
$$

Then, the following existence theorem is a special case of the result in [14].

Theorem 3.1. Let $\mathcal{A}$ and $\mathcal{B}$ be as described above. Then, for each $\mathbf{w}_{0} \in G$ and $l \in \mathbb{F}^{\prime}$ there exists a $\mathbf{w} \in \mathbb{X}$ satisfying

$$
\begin{gathered}
(\mathcal{B} \mathbf{w})^{\prime}+\mathcal{A} \mathbf{w}=l \quad \text { in } \mathbb{F}^{\prime} \\
\mathcal{B} \mathbf{w}(0)=\mathcal{B} \mathbf{w}_{0} \quad \text { in } G^{\prime} .
\end{gathered}
$$

We turn to describe the sequence of regularized problems whose solutions will be given by Theorem 3.1. To replace the inclusion in (2.29) with an equality we regularize the norm function on $\mathbb{R}^{m}$. Let $\left(\psi^{h}\right)_{h>0}$ be a family of smooth approximations to $|\cdot|$ such that, for each $h>0, \psi^{h} \in C^{1}\left(\mathbb{R}^{m}\right)$ is positive, convex and

$$
\left|\nabla \psi^{h}(s)\right| \leq 2, \quad 0 \leq\left\langle\nabla \psi^{h}(s), s\right\rangle, \quad \text { and } \quad\left|\psi^{h}(s)-\right| s|| \leq h,
$$

for all $s \in \mathbb{R}^{m}$. We define the operator $J^{h}: E \rightarrow E^{\prime}$ by

$$
\left\langle J^{h} v, w\right\rangle=\int_{\Gamma_{C}} \mu\left|R g_{n}\right|\left(1-\delta\left|R g_{n}\right|\right)_{+} \nabla \psi^{h}\left(v_{\tau}-\phi\right) \cdot w_{\tau} d \Gamma .
$$

We let $R_{e}: E \rightarrow E^{\prime}$ denote the Riesz map, and for the sake of simplicity, we will omit the subscript $g$ below.

We now consider, for each $h>0$, the following regularized problem. 
Problem $P_{h}$ : Find a triplet $\left\{\mathbf{u}_{h}, \mathbf{v}_{h}, \xi_{h}\right\}$ satisfying

$$
\begin{aligned}
& \xi_{h} \in \mathbb{V}, \quad \xi_{h}^{\prime} \in \mathbb{V}^{\prime}, \quad \mathbf{u}_{h} \in \mathbb{E}, \quad \mathbf{v}_{h} \in \mathbb{E}, \\
& \xi_{h}^{\prime}+K_{1} \xi_{h}+K_{2} \xi_{h}+C_{1} \mathbf{v}_{h}+S\left(g, \mathbf{v}_{h}\right)=Q \quad \text { in } \mathbb{V}^{\prime}, \\
& B \mathbf{v}_{h}+A \mathbf{u}_{h}+C_{2} \xi_{h}+J^{h} \mathbf{v}_{h}=f \quad \text { in } \mathbb{E}^{\prime}, \\
& \left(R_{e} \mathbf{u}_{h}\right)^{\prime}-R_{e} \mathbf{v}_{h}=0 \quad \text { in } \mathbb{E}^{\prime},
\end{aligned}
$$

with the initial conditions

$$
\mathbf{u}_{h}(0)=\mathbf{u}_{0}, \quad \boldsymbol{\xi}_{h}(0)=\boldsymbol{\xi}_{0} .
$$

We have the following existence result for this problem.

Theorem 3.2. Let $h>0$. Then there exists a solution for Problem $P_{h}$.

Proof. We fit Problem $P_{h}$ into the framework of Theorem 3.1 by choosing $F=V \times$ $E \times E$ and $G=H \times H^{m} \times E, \mathbf{w} \in G, \mathbf{w}_{0} \in G$ and $l \in \mathbb{F}^{\prime}$ given by

$$
\mathbf{w}=\left(\begin{array}{c}
\xi \\
\mathbf{v} \\
\mathbf{u}
\end{array}\right), \quad \mathbf{w}_{0}=\left(\begin{array}{c}
\xi_{0} \\
\mathbf{v}_{0} \\
\mathbf{u}_{0}
\end{array}\right), \quad l=\left(\begin{array}{c}
Q \\
f \\
0
\end{array}\right) .
$$

The operators $\mathcal{B}: G \rightarrow G^{\prime}$ and $\mathcal{A}(t, \cdot): F \rightarrow F^{\prime}$ are chosen as

$$
\mathcal{B} \mathbf{w}=\left(\begin{array}{c}
\xi \\
0 \\
R_{e} \mathbf{u}
\end{array}\right), \quad \mathcal{A}(t, \mathbf{w})=\left(\begin{array}{c}
K_{1} \xi+K_{2} \xi+C_{1} \mathbf{v}+S(g, \mathbf{v}) \\
B \mathbf{v}+A \mathbf{u}+C_{2} \xi+J^{h} \mathbf{v} \\
-R_{e} \mathbf{v}
\end{array}\right)
$$

Now, the verification of the assumptions of Theorem 3.1 is routine; so we establish only the pseudomonotonicity (3.1) and the coercivity (3.3) conditions. In checking the pseudomonotonicity of the operator $\mathcal{A}$, we use the fact that we may write $\mathcal{A}=\mathcal{A}_{1}+\mathcal{A}_{2}$, where

$$
\mathcal{A}_{1}(t, \mathbf{w})=\left(\begin{array}{c}
K_{1} \xi+K_{2} \xi+C_{1} \mathbf{v} \\
B \mathbf{v}+A \mathbf{u}+C_{2} \xi \\
-R_{e} \mathbf{v}
\end{array}\right)
$$

gives rise to a bounded linear operator from $\mathbb{X}$ to $\mathbb{X}^{\prime}$ (hence weakly continuous and thus pseudomonotone [6]), and

$$
\mathcal{A}_{2}(t, \mathbf{w})=\left(\begin{array}{c}
S(g, \mathbf{v}) \\
J^{h} \mathbf{v} \\
0
\end{array}\right)
$$

is a weak to norm continuous operator from $\mathbb{X}$ to $\mathbb{X}^{\prime}$. To establish the latter property we recall that $\mathbb{X}=\left\{w \in \mathbb{F}:(\mathcal{B} w)^{\prime} \in \mathbb{F}^{\prime}\right\}$, and employ the following lemma.

LEMma 3.3. Let $\mathbb{Y}=\left\{u \in \mathbb{E}:\left(R_{e} u\right)^{\prime} \in \mathbb{E}^{\prime}\right\}$ with the graph norm $\|u\|_{\mathbb{Y}}=\|u\|_{\mathbb{E}}+$ $\left\|\left(R_{e} u\right)^{\prime}\right\|_{\mathbb{E}^{\prime}}$, and let $S(g, \cdot): \mathbb{Y} \rightarrow \mathbb{V}^{\prime}$ and $J^{h}: \mathbb{Y} \rightarrow \mathbb{Y}^{\prime}$ be given by (2.27) and (3.4), respectively. Then $S(g, \cdot)$ and $J^{h}$ are weak to norm continuous. 
Proof. We establish the result only for the operator $S(g, \cdot)$ since the proof for $J^{h}$ is similar. It is enough to show that every subsequence of a weakly convergent sequence $\left\{v_{k}\right\}$, which converges to $v$ in $\mathbb{Y}$, has a further subsequence whose images under $S(g, \cdot)$ converge to $S(g, v)$. So let $\left\{v_{k_{j}}\right\}$ be a subsequence of $\left\{v_{k}\right\}$. Since $\left\{v_{k_{j}}\right\}$ is bounded in $\mathbb{Y}$ and since the injection $H^{1}(\Omega)^{m} \rightarrow H^{1-\varepsilon}(\Omega)^{m}$ is compact for any $\varepsilon>0([6])$, we have by a theorem of Lions [19], that $\left\{v_{k_{j}}\right\}$ is relatively compact in $L^{2}\left(0, T ; H^{1-\varepsilon}(\Omega)^{m}\right)$. By the continuity of the trace map $L^{2}\left(0, T ; H^{1-\varepsilon}(\Omega)^{m}\right) \rightarrow L^{2}\left(0, T ; L^{2}(\Gamma)^{m}\right)=\left(L^{2}(\Gamma \times(0, T))\right)^{m}$ we may, upon passing to a subsequence denoted also by $\left\{v_{k_{j}}\right\}$, suppose that $v_{k_{j}}(x, t) \rightarrow$ $v(x, t)$ for almost all $(x, t) \in \Gamma_{C} \times(0, T)$. Since $s_{c}(\cdot, r) \leq \alpha_{4}$ and $\left|R g_{n}\right|\left(1-\delta\left|R g_{n}\right|\right)_{+} \leq \frac{1}{\delta}$ we obtain that $\left\{\left|R g_{n}\right|\left(1-\delta\left|R g_{n}\right|\right)_{+} s_{c}\left(\cdot,\left|\left(v_{k_{j}}\right)_{\tau}-\phi\right|\right)\right\}$ is a bounded sequence in $L^{2}\left(\Gamma_{C} \times\right.$ $(0, T))$. Consequently, it converges to $\left|R g_{n}\right|\left(1-\delta\left|R g_{n}\right|\right)_{+} s_{c}\left(\cdot,\left|v_{\tau}-\phi\right|\right)$ in $L^{2}\left(\Gamma_{C} \times(0, T)\right)$. Finally, using Cauchy's inequality, we deduce that $S\left(g, v_{k_{j}}\right) \rightarrow S(g, v)$ in $\mathbb{Y}^{\prime}$ when $j \rightarrow \infty$. This completes the proof.

In checking the coercivity condition (3.3), we estimate various terms using Cauchy's inequality, standard trace theorems and the foliowing result. Here and below $c$ represents a positive constant whose value may change from line to line, but in all cases depends only on the data and coefficients of the approximate problem.

Lemma 3.4. Assume that (2.11)-(2.15) hold. Then there exists a positive constant $\alpha$ such that

$$
\langle A u, u\rangle \geq \alpha\|u\|_{E}^{2}, \quad\langle B v, v\rangle \geq \alpha\|v\|_{E}^{2}, \quad\left\langle K_{2} \xi, \xi\right\rangle \geq \alpha\|\xi\|_{V}^{2}
$$

and also

$$
\left\langle J^{h} v, v\right\rangle \geq-c
$$

Proof. The first three inequalities are consequences of assumptions (2.11) and Korn's inequality. The last estimate is a result of the following decomposition:

$$
\begin{aligned}
\left\langle J^{h} v, v\right\rangle=\int_{\Gamma_{C}} \mu\left|R g_{n}\right|\left(1-\delta\left|R g_{n}\right|\right)_{+} \nabla \psi^{h}( & \left.v_{\tau}-\phi\right) \cdot\left(v_{\tau}-\phi\right) d \Gamma \\
& +\int_{\Gamma_{C}} \mu\left|R g_{n}\right|\left(1-\delta\left|R g_{n}\right|\right)_{+} \nabla \psi^{h}\left(v_{\tau}-\phi\right) \cdot \phi d \Gamma
\end{aligned}
$$

in which the first integral is nonnegative and the second one is bounded. This concludes the proof of the lemma and of Theorem 3.2.

The next step in the proof of Theorem 2.3 deals with an a priori estimate on the solutions of Problem $P_{h}$.

Theorem 3.5. Let $\left\{\mathbf{u}_{h}, \mathbf{v}_{h}, \xi_{h}\right\}$ be a solution of Problem $P_{h}$ corresponding to the parameter $h$. Then there exists a positive constant $c$, independent of $h$, such that, for all $t \in[0, T]$,

$$
\left\|\mathbf{u}_{h}(t)\right\|_{E}^{2}+\int_{0}^{t}\left\|\mathbf{v}_{h}(s)\right\|_{E}^{2} d s+\left|\xi_{h}(t)\right|_{H}^{2}+\int_{0}^{t}\left\|\xi_{h}(s)\right\|_{V}^{2} d s \leq c .
$$


Proof. For the sake of simplicity, we will omit the subscript $h$ below. We begin by letting (3.7) act on $\mathbf{v}$. Thus,

$$
\int_{0}^{t}\langle B \mathbf{v}, \mathbf{v}\rangle d s+\int_{0}^{t}\langle A \mathbf{u}, \mathbf{v}\rangle d s+\int_{0}^{t}\left\langle J^{h} \mathbf{v}, \mathbf{v}\right\rangle d s=-\int_{0}^{t}\left\langle C_{2} \xi, \mathbf{v}\right\rangle d s+\int_{0}^{t}\langle f, \mathbf{v}\rangle d s
$$

We will now estimate each of the terms in (3.13). Applying Lemma 3.4 yields

$$
\begin{aligned}
\int_{0}^{t}\langle B \mathbf{v}, \mathbf{v}\rangle d s \geq \alpha \int_{0}^{t}\|\mathbf{v}(s)\|_{E}^{2} d s \\
\int_{0}^{t}\langle A \mathbf{u}, \mathbf{v}\rangle d s=\frac{1}{2} \int_{0}^{t}\left(\frac{d}{d s}\langle A \mathbf{u}, \mathbf{u}\rangle\right) d s=\frac{1}{2}\langle A \mathbf{u}(t), \mathbf{u}(t)\rangle-\frac{1}{2}\left\langle A \mathbf{u}_{0}, \mathbf{u}_{0}\right\rangle \\
\geq \frac{\alpha}{2}\|\mathbf{u}(t)\|_{E}^{2}-c\left\|\mathbf{u}_{0}\right\|_{E}^{2},
\end{aligned}
$$

and

$$
\int_{0}^{t}\left\langle J^{h} \mathbf{v}, \mathbf{v}\right\rangle d s \geq-c
$$

The integrals on the right in (3.13) are estimated by using Cauchy's inequality with $\varepsilon$,

$$
\begin{aligned}
\left|\int_{0}^{t}\left\langle C_{2} \xi, \mathbf{v}\right\rangle d s\right| & \leq c \int_{0}^{t}|\xi(s)|_{H}^{2} d s+\frac{\alpha}{4} \int_{0}^{t}\|\mathbf{v}(s)\|_{E}^{2} d s \\
\left|\int_{0}^{t}\langle f, \mathbf{v}\rangle d s\right| & \leq c \int_{0}^{t}\|f(s)\|_{E^{\prime}}^{2} d s+\frac{\alpha}{4} \int_{0}^{t}\|\mathbf{v}(s)\|_{E}^{2} d s
\end{aligned}
$$

Combining the estimates (3.14)-(3.18) in (3.13), we find

$$
\|\mathbf{u}(t)\|_{E}^{2}+\int_{0}^{t}\|\mathbf{v}(s)\|_{E}^{2} d s \leq c\left(1+\int_{0}^{t}|\xi(s)|_{H}^{2} d s\right) .
$$

We turn now to the energy equation (3.6) and by letting it act on $\xi$ we obtain

$$
\begin{aligned}
\int_{0}^{t}\left\langle\xi^{\prime}, \xi\right\rangle d s+\int_{0}^{t}\left\langle K_{1} \xi, \xi\right\rangle d s+\int_{0}^{t}\left\langle K_{2} \xi, \xi\right\rangle d s \\
=-\int_{0}^{t}\left\langle C_{1} \mathbf{v}, \xi\right\rangle d s-\int_{0}^{t}\langle S(g, \mathbf{v}), \xi\rangle d s+\int_{0}^{t}\langle Q, \xi\rangle d s
\end{aligned}
$$

From Theorem 1(2) in [14] and our choice of the spaces $F$ and $G$ we find

$$
\int_{0}^{t}\left\langle\xi^{\prime}, \xi\right\rangle d s=\frac{1}{2} \int_{0}^{t}\left(\frac{d}{d s}|\xi(s)|_{H}^{2}\right) d s=\frac{1}{2}|\xi(t)|_{H}^{2}-\frac{1}{2}\left|\xi_{0}\right|_{H}^{2}
$$


Moreover, using arguments similar to those above (see (3.14), (3.17), and (3.18)) we get

$$
\begin{gathered}
\int_{0}^{t}\left\langle K_{1} \xi, \xi\right\rangle d s=\int_{0}^{t} \int_{\Gamma_{C}} k_{e}|\xi|^{2} d s \geq 0 \\
\int_{0}^{t}\left\langle K_{2} \xi, \xi\right\rangle d s \geq \alpha \int_{0}^{t}\|\xi\|_{V}^{2} d s \\
\left|\int_{0}^{t}\left\langle C_{1} \mathbf{v}, \xi\right\rangle d s\right| \leq c \int_{0}^{t}|\mathbf{v}(s)|_{H^{m}}^{2} d s+\frac{\alpha}{4} \int_{0}^{t}\|\xi(s)\|_{V}^{2} d s \\
\left|\int_{0}^{t}\langle Q, \xi\rangle d s\right| \leq c \int_{0}^{t}\|Q(s)\|_{V^{\prime}}^{2} d s+\frac{\alpha}{4} \int_{0}^{t}\|\xi(s)\|_{V}^{2} d s
\end{gathered}
$$

To estimate the term involving $S(g, \cdot)$, we use the inequality $\left|R g_{n}\right|\left(1-\delta\left|R g_{n}\right|\right)_{+} \leq \frac{1}{\delta}$, assumption (2.14), and Cauchy's inequality with $\varepsilon$, and deduce that

$$
\left|\int_{0}^{t}\langle S(g, \mathbf{v}), \xi\rangle d s\right| \leq c+\frac{\alpha}{4} \int_{0}^{t}\|\xi(s)\|_{V}^{2} d s .
$$

Combining the estimates (3.21)-(3.26) in (3.20) we obtain

$$
|\xi(t)|_{H}^{2}+\int_{0}^{t}\|\xi(s)\|_{V}^{2} d s \leq c,
$$

and thus,

$$
\int_{0}^{t}|\xi(s)|_{H}^{2} d s \leq c .
$$

Inserting this estimate in (3.19) we deduce that

$$
\|\mathbf{u}(t)\|_{E}^{2}+\int_{0}^{t}\|\mathbf{v}(s)\|_{E}^{2} d s \leq c .
$$

The estimate (3.12) is now a consequence of (3.27) and (3.28).

4. Proof of Theorem 2.3. We prove the existence of the unique solution of Problem $P_{g}$ as a limit of a subsequence of solutions for Problem $P_{h}$ obtained above. It follows from Theorem 3.5 that for a given set of initial conditions the family of solutions $\left\{\mathbf{u}_{h}, \mathbf{v}_{h}, \xi_{h}\right\}$ is bounded in $\mathbb{E} \times \mathbb{E} \times \mathbb{V}$. From this and (3.6)-(3.8) it follows that $\left\{R_{e} \mathbf{u}_{h}^{\prime}, \xi_{h}^{\prime}\right\}$ is bounded in $\mathbb{E}^{\prime} \times \mathbb{V}^{\prime}$. Consequently, there exists a weak limit point $(\mathbf{u}, \mathbf{v}, \xi) \in \mathbb{E} \times \mathbb{E} \times \mathbb{V}$ and a subsequence of parameters $\left\{h_{l}\right\}$ such that $h_{l} \rightarrow 0$ when $l \rightarrow \infty$ and such that the following limit processes take place when $l \rightarrow \infty$ :

$$
\begin{array}{rlrl}
\mathbf{u}_{l} & \rightarrow \mathbf{u} & & \text { weakly in } \mathbb{E}, \\
\mathbf{v}_{l} & \rightarrow \mathbf{v} & \text { weakly in } \mathbb{E}, \\
\xi_{l} & \rightarrow \xi & \text { weakly in } \mathbb{V}, \\
\xi_{l}^{\prime} & \rightarrow \xi^{\prime} & & \text { weakly in } \mathbb{V}^{\prime}, \\
R_{e} \mathbf{u}_{l}^{\prime} & \rightarrow R_{e} \mathbf{u}^{\prime} & & \text { weakly in } \mathbb{E}^{\prime} .
\end{array}
$$

We may pass now to the limit in (3.6) and (3.7) in all terms except the one involving $J^{h_{l}}$. We may suppose, by passing to a subsequence if necessary, that $J^{h_{l}}\left(\mathbf{v}_{l}\right) \rightarrow \gamma$ weakly 
in $\mathbb{E}^{\prime}$, for some $\gamma \in \mathbb{E}^{\prime}$. It only remains to show that $\gamma \in \partial_{2} j(g, \mathbf{v})$. Toward that end, we note that when $l \rightarrow \infty$ we have

$$
\begin{gathered}
\psi^{h_{l}}\left(w_{\tau}-\mathbf{v}_{\tau}+\left(\mathbf{v}_{l}\right)_{\tau}-\phi\right) \rightarrow\left|w_{\tau}-\phi\right| \quad \text { in } L^{2}\left(\Gamma_{C} \times(0, T)\right), \\
\psi^{h_{l}}\left(\left(\mathbf{v}_{l}\right)_{\tau}-\phi\right) \rightarrow\left|\mathbf{v}_{\tau}-\phi\right| \quad \text { in } L^{2}\left(\Gamma_{C} \times(0, T)\right),
\end{gathered}
$$

for $w \in \mathbb{E}$. Then, it follows from the convexity of $\psi^{h_{l}}$ and Hölder's inequality that

$$
\begin{aligned}
\langle\gamma, w-\mathbf{v}\rangle= & \lim _{l \rightarrow \infty}\left\langle J^{h_{l}} \mathbf{v}_{l}, w-\mathbf{v}\right\rangle \\
= & \lim _{l \rightarrow \infty} \int_{0}^{T} \int_{\Gamma_{C}} \mu\left|R g_{n}\right|\left(1-\delta\left|R g_{n}\right|\right)_{+} \nabla \psi^{h_{l}}\left(\left(\mathbf{v}_{l}\right)_{\tau}-\phi\right) \cdot\left(w_{\tau}-\mathbf{v}_{\tau}\right) d \Gamma d t \\
\leq & \lim _{l \rightarrow \infty} \int_{0}^{T} \int_{\Gamma_{C}} \mu\left|R g_{n}\right|\left(1-\delta\left|R g_{n}\right|\right)_{+} \\
& \times\left[\psi^{h_{l}}\left(w_{\tau}-\mathbf{v}_{\tau}+\left(\mathbf{v}_{l}\right)_{\tau}-\phi\right)-\psi^{h_{l}}\left(\left(\mathbf{v}_{l}\right)_{\tau}-\phi\right)\right] d \Gamma d t \\
\leq & \int_{0}^{T} \int_{\Gamma_{C}} \mu\left|R g_{n}\right|\left(1-\delta\left|R g_{n}\right|\right)_{+}\left[\left|w_{\tau}-\phi\right|-\left|\mathbf{v}_{\tau}-\phi\right|\right] d \Gamma d t .
\end{aligned}
$$

This shows that $\gamma \in \partial_{2} j(g, \mathbf{v})$. Consequently, the weak limit $\{\mathbf{u}, \xi\}$ of the subsequence $\left\{\mathbf{u}_{l}, \xi_{l}\right\}$ is a solution to $(2.30),(2.31),(2.33),(2.34)$. Let now $\sigma$ be given by $\sigma_{i j}=$ $a_{i j k l} u_{k, l}+b_{i j k l} u_{k, l}^{\prime}-c_{i j}(\xi-\Theta)$. To show that $\sigma \in \mathbb{W}$ we let $(2.34)$ act on $\varphi \in \mathcal{D}(\Omega)^{m}$ followed by Green's formula which implies that $\sigma_{i j, j}+f_{A i}=0$. This completes the proof of the existence part in Theorem 2.3.

We will prove now that the solution of Problem $P_{g}$ is unique. Indeed, let $\left\{\mathbf{u}_{1}, \sigma_{1}, \xi_{1}\right\}$ and $\left\{\mathbf{u}_{2}, \sigma_{2}, \xi_{2}\right\}$ be two solutions corresponding to the same data. Taking (2.32) into account, it suffices to show that $\mathbf{u}_{1}=\mathbf{u}_{2}$ and $\xi_{1}=\xi_{2}$. To this end, we substitute into (2.34) $\mathbf{u}_{1}$ for $\mathbf{u}$ and let the resulting expression act on $\mathbf{u}_{1}^{\prime}-\mathbf{u}_{2}^{\prime}$ and then substitute into (2.34) $\mathbf{u}_{2}$ for $\mathbf{u}$ and let the resulting expression act on $\mathbf{u}_{2}^{\prime}-\mathbf{u}_{1}^{\prime}$. Adding the two inequalities and using the notation $\mathbf{u}=\mathbf{u}_{1}-\mathbf{u}_{2}, \mathbf{v}=\mathbf{u}_{1}^{\prime}-\mathbf{u}_{2}^{\prime}$, and $\xi=\xi_{1}-\xi_{2}$ yields

$$
\int_{0}^{t}\langle B \mathbf{v}, \mathbf{v}\rangle d s+\int_{0}^{t}\langle A \mathbf{u}, \mathbf{v}\rangle d s \leq-\int_{0}^{t}\left\langle C_{2} \xi, \mathbf{v}\right\rangle d s .
$$

We estimate the terms in (4.1) using the same arguments as those used to estimate the corresponding terms in (3.13) (see (3.14), (3.15), and (3.17)). Combining the resulting estimates in (4.1), we find

$$
\|\mathbf{u}(t)\|_{E}^{2}+\int_{0}^{t}\|\mathbf{v}(s)\|_{E}^{2} d s \leq c \int_{0}^{t}|\xi(s)|_{H}^{2} d s .
$$

Next, we substitute $\xi_{1}$ and $\xi_{2}$ into (2.33) in turn, subtract the resulting equations and let the result act on $\xi$. We obtain

$$
\begin{aligned}
\int_{0}^{t}\left\langle\xi^{\prime}, \xi\right\rangle d s+\int_{0}^{t}\left\langle K_{1} \xi, \xi\right\rangle d s+ & \int_{0}^{t}\left\langle K_{2} \xi, \xi\right\rangle d s \\
& =-\int_{0}^{t}\left\langle C_{1} \mathbf{v}, \xi\right\rangle d s-\int_{0}^{t}\left\langle S\left(g, \mathbf{v}_{1}\right)-S\left(g, \mathbf{v}_{2}\right), \xi\right\rangle d s
\end{aligned}
$$


All the terms in this equality, except the last one, can be estimated in the same way as (3.21)-(3.24). To estimate the term involving $S(g, \cdot)$ we use $(2.27)$ and get

$$
\begin{aligned}
& \left|\int_{0}^{t}\left\langle S\left(g, \mathbf{v}_{1}\right)-S\left(g, \mathbf{v}_{2}\right), \xi\right\rangle d s\right| \\
& \quad \leq \int_{0}^{t} \int_{\Gamma_{C}} \mu\left|R g_{n}\right|\left(1-\delta\left|R g_{n}\right|\right)_{+}\left|s_{c}\left(x,\left|\left(\mathbf{v}_{1}\right)_{\tau}-\phi\right|\right)-s_{c}\left(x,\left|\left(\mathbf{v}_{2}\right)_{\tau}-\phi\right|\right)\right||\xi| d \Gamma d s .
\end{aligned}
$$

Since $\left|R g_{n}\right|\left(1-\delta\left|R g_{n}\right|\right)_{+} \leq \frac{1}{\delta}$, by using (2.14) followed by Cauchy's inequality with $\varepsilon$, we obtain

$$
\left|\int_{0}^{t}\left\langle S\left(g, \mathbf{v}_{1}\right)-S\left(g, \mathbf{v}_{2}\right), \xi\right\rangle d s\right| \leq c \int_{0}^{t}\|\mathbf{v}(s)\|_{E}^{2} d s+\frac{\alpha}{4} \int_{0}^{t}\|\xi(s)\|_{V}^{2} d s .
$$

Here we used in an essential way the assumption of SJK. Combining these estimates in (4.3) we get

$$
|\xi(t)|_{H}^{2}+\int_{0}^{t}\|\xi(s)\|_{V}^{2} d s \leq c \int_{0}^{t}\|\mathbf{v}(s)\|_{E}^{2} d s .
$$

We deduce from (4.2) that

$$
\int_{0}^{t}\|\mathbf{v}(s)\|_{E}^{2} d s \leq c \int_{0}^{t}|\xi(s)|_{H}^{2} d s
$$

which, when used in (4.4), implies that

$$
|\xi(t)|_{H}^{2} \leq c \int_{0}^{t}|\xi(s)|_{H}^{2} d s
$$

Now, it follows from Gronwall's inequality that $\xi(t)=0$, and then (4.2) implies that $\mathbf{u}(t)=0$. This completes the proof of Theorem 2.3.

REMARK 4.1. The arguments of this section may be used to show that the solution of each of the approximate problems $P_{h}$ is unique, too.

5. Proof of Theorem 2.2. We use a fixed point argument to establish the existence of a unique solution for Problem $P$ when $|\mu|_{L^{\infty}\left(\Gamma_{C}\right)}$ is sufficiently small. Theorem 2.3 asserts that for each $g \in \mathbb{W}$, Problem $P_{g}$ has a unique solution $\left\{\mathbf{u}_{g}, \sigma_{g}, \xi_{g}\right\}$. Consequently, we consider the operator $\Lambda: \mathbb{W} \rightarrow \mathbb{W}$ defined by

$$
\Lambda g=\sigma_{g}, \quad g \in \mathbb{W} .
$$

We have

Proposition 5.1. There exists $\mu_{0}>0$, sufficiently small, such that if $|\mu|_{L^{\infty}\left(\Gamma_{C}\right)}<\mu_{0}$, then the operator $\Lambda$ has a unique fixed point $g^{*} \in \mathbb{W}$.

Proof. Let $g^{1}, g^{2} \in \mathbb{W}$ and set $g=g^{1}-g^{2}, \mathbf{u}^{i}=\mathbf{u}_{g^{i}}, \mathbf{v}^{i}=\mathbf{u}_{g^{i}}^{\prime}, \sigma^{i}=\sigma_{g^{i}}$, and $\xi^{i}=\xi_{g^{i}}$, where $i=1$ or 2 . We substitute into (2.34) $\mathbf{u}^{1}$ for $\mathbf{u}$ and let the resulting expression act on $\mathbf{v}^{1}-\mathbf{v}^{2}$ and then substitute into (2.34) $\mathbf{u}^{2}$ for $\mathbf{u}$ and let the resulting expression 
act on $\mathbf{v}^{2}-\mathbf{v}^{1}$. We add the two inequalities, denote by $\mathbf{u}=\mathbf{u}^{1}-\mathbf{u}^{2}, \mathbf{v}=\mathbf{v}^{1}-\mathbf{v}^{2}$, and $\xi=\xi^{1}-\xi^{2}$, and obtain

$$
\begin{aligned}
\int_{0}^{t}\langle B \mathbf{v}, \mathbf{v}\rangle d s+ & \int_{0}^{t}\langle A \mathbf{u}, \mathbf{v}\rangle d s+\int_{0}^{t}\left\langle C_{2} \xi, \mathbf{v}\right\rangle d s \\
& \leq \int_{0}^{t} \int_{\Gamma_{C}} \mu\left(\left|R g_{n}^{1}\right|\left(1-\delta\left|R g_{n}^{1}\right|\right)_{+}-\left|R g_{n}^{2}\right|\left(1-\delta\left|R g_{n}^{2}\right|\right)_{+}\right) \\
& \quad \times\left(\left|\left(\mathbf{v}_{1}\right)_{\tau}-\phi\right|-\left|\left(\mathbf{v}_{2}\right)_{\tau}-\phi\right|\right) d \Gamma d s .
\end{aligned}
$$

We estimate the terms on the left-hand side in (5.2) by the same arguments as those used to estimate the corresponding terms in (3.13) (see (3.14), (3.15), and (3.17)). The integral on the right-hand side is estimated by using the inequality

$$
\left|R g_{n}^{1}\right|\left(1-\delta\left|R g_{n}^{1}\right|\right)_{+}-\left|R g_{n}^{2}\right|\left(1-\delta\left|R g_{n}^{2}\right|\right)_{+} \leq c\left|R g_{n}^{1}-R g_{n}^{2}\right|
$$

followed by Cauchy's inequality with $\varepsilon$, to obtain

$$
\begin{array}{r}
\int_{0}^{t} \int_{\Gamma_{C}} \mu\left(\left|R g_{n}^{1}\right|\left(1-\delta\left|R g_{n}^{1}\right|\right)_{+}-\left|R g_{n}^{2}\right|\left(1-\delta\left|R g_{n}^{2}\right|\right)_{+}\right)\left(\left|\left(\mathbf{v}_{1}\right)_{\tau}-\phi\right|-\left|\left(\mathbf{v}_{2}\right)_{\tau}-\phi\right|\right) d \Gamma d s \\
\leq c|\mu|_{L^{\infty}\left(\Gamma_{C}\right)}^{2} \int_{0}^{t}\left|R g_{n}^{1}(s)-R g_{n}^{2}(s)\right|_{L^{2}(\Gamma)}^{2} d s+\frac{\alpha}{4} \int_{0}^{t}\|\mathbf{v}(s)\|_{E}^{2} d s
\end{array}
$$

Combining these estimates in (5.2), we get

$$
\|\mathbf{u}(t)\|_{E}^{2}+\int_{0}^{t}\|\mathbf{v}(s)\|_{E}^{2} d s \leq c \int_{0}^{t}|\xi(s)|_{H}^{2} d s+c|\mu|_{L^{\infty}\left(\Gamma_{C}\right)}^{2} \int_{0}^{t}\left|R g_{n}^{1}(s)-R g_{n}^{2}(s)\right|_{L^{2}(\Gamma)}^{2} d s,
$$

which implies

$$
\|\mathbf{u}(t)\|_{E}^{2}+\int_{0}^{t}\|\mathbf{v}(s)\|_{E}^{2} d s \leq c \int_{0}^{t}|\xi(s)|_{H}^{2} d s+c|\mu|_{L^{\infty}\left(\Gamma_{C}\right)}^{2} \int_{0}^{t}\|g(s)\|_{W}^{2} d s .
$$

Next, we substitute $\xi_{1}$ and $\xi_{2}$ into (2.33) in turn, subtract the resulting equations and let the result act on the difference $\xi$ and obtain

$$
\begin{aligned}
\int_{0}^{t}\left\langle\xi^{\prime}, \xi\right\rangle d s+\int_{0}^{t}\left\langle K_{1} \xi, \xi\right\rangle d s & +\int_{0}^{t}\left\langle K_{2} \xi, \xi\right\rangle d s \\
& =-\int_{0}^{t}\left\langle C_{1} \mathbf{v}, \xi\right\rangle d s-\int_{0}^{t}\left\langle S\left(g^{1}, \mathbf{v}^{1}\right)-S\left(g^{2}, \mathbf{v}^{2}\right), \xi\right\rangle d s .
\end{aligned}
$$

All the terms in this equality, except the last one, can be estimated in the same way as (3.21)-(3.24). To estimate the term involving $S$ we write

$$
\begin{aligned}
\int_{0}^{t}\left\langle S\left(g^{2}, \mathbf{v}^{2}\right)-S\left(g^{1}\right.\right. & \left.\left., \mathbf{v}^{1}\right), \xi\right\rangle d s \\
& \leq \int_{0}^{t}\left\langle S\left(g^{2}, \mathbf{v}^{1}\right)-S\left(g^{1}, \mathbf{v}^{1}\right), \xi\right\rangle d s+\int_{0}^{t}\left\langle S\left(g^{2}, \mathbf{v}^{2}\right)-S\left(g^{2}, \mathbf{v}^{1}\right), \xi\right\rangle d s .
\end{aligned}
$$

Using (2.27), (5.3), and (2.14), the first integral on the right-hand side is estimated by Cauchy's inequality with $\varepsilon$ thus,

$$
\int_{0}^{t}\left\langle S\left(g^{2}, \mathbf{v}^{1}\right)-S\left(g^{1}, \mathbf{v}^{1}\right), \xi\right\rangle d s \leq c|\mu|_{L^{\infty}\left(\Gamma_{C}\right)}^{2} \int_{0}^{t}\|g(s)\|_{W}^{2} d s+\frac{\alpha}{4} \int_{0}^{t}\|\xi(s)\|_{V}^{2} d s .
$$


Using (2.27), (2.14), and the inequality $\left|R g_{n}\right|\left(1-\delta\left|R g_{n}\right|\right)_{+} \leq \frac{1}{\delta}$, we find that the second integral on the right-hand side satisfies

$$
\left|\int_{0}^{t}\left\langle S\left(g^{2}, \mathbf{v}^{2}\right)-S\left(g^{2}, \mathbf{v}^{1}\right), \xi\right\rangle d s\right| \leq c \int_{0}^{t}\|\mathbf{v}(s)\|_{E}^{2} d s+\frac{\alpha}{4} \int_{0}^{t}\|\xi(s)\|_{V}^{2} d s .
$$

Combining these estimates in (5.5) we obtain

$$
|\xi(t)|_{H}^{2}+\int_{0}^{t}\|\xi(s)\|_{V}^{2} d s \leq c \int_{0}^{t}\|\mathbf{v}(s)\|_{E}^{2} d s+c|\mu|_{L^{\infty}\left(\Gamma_{C}\right)}^{2} \int_{0}^{t}\|g(s)\|_{W}^{2} d s .
$$

We deduce from (4.4) that

$$
\int_{0}^{t}\|\mathbf{v}(s)\|_{E}^{2} d s \leq c \int_{0}^{t}|\xi(s)|_{H}^{2} d s+c|\mu|_{L^{\infty}\left(\Gamma_{C}\right)}^{2} \int_{0}^{t}\|g(s)\|_{W}^{2} d s,
$$

which, when used in (5.6), yields

$$
|\xi(t)|_{H}^{2} \leq c \int_{0}^{t}|\xi(s)|_{H}^{2} d s+c|\mu|_{L^{\infty}\left(\Gamma_{C}\right)}^{2} \int_{0}^{t}\|g(s)\|_{W}^{2} d s .
$$

By using Gronwall's inequality we obtain

$$
|\xi(t)|_{H}^{2} \leq c|\mu|_{L^{\infty}\left(\Gamma_{C}\right)}^{2} \int_{0}^{t}\|g(s)\|_{W}^{2} d s
$$

Applying this estimate in (5.4), we find

$$
\begin{array}{r}
\|\mathbf{u}(t)\|_{E}^{2} \leq c|\mu|_{L^{\infty}\left(\Gamma_{C}\right)}^{2}\left(\int_{0}^{t} \int_{0}^{s}\|g(r)\|_{W}^{2} d r d s+\int_{0}^{t}\|g(s)\|_{W}^{2} d s\right), \\
\int_{0}^{t}\|\mathbf{v}(s)\|_{E}^{2} d s \leq c|\mu|_{L^{\infty}\left(\Gamma_{C}\right)}^{2}\left(\int_{0}^{t} \int_{0}^{s}\|g(r)\|_{W}^{2} d r d s+\int_{0}^{t}\|g(s)\|_{W}^{2} d s\right) .
\end{array}
$$

We conclude, using (5.8)-(5.10), that

$$
\|\mathbf{u}\|_{\mathbb{E}}^{2}+\|\mathbf{v}\|_{\mathbb{E}}^{2}+|\xi|_{\mathbb{H}}^{2} \leq c|\mu|_{L^{\infty}\left(\Gamma_{C}\right)}^{2}\|g\|_{\mathbb{W}}^{2} .
$$

On the other hand, it follows from (2.32) and (2.11) that

$$
\left\|\sigma^{1}-\sigma^{2}\right\|_{\mathbb{W}}^{2} \leq c\|\mathbf{u}\|_{\mathbb{E}}^{2}+\|\mathbf{v}\|_{\mathbb{E}}^{2}+|\xi|_{\mathbb{H}}^{2} .
$$

Combining the estimates (5.11) and (5.12) we find

$$
\left\|\sigma^{1}-\sigma^{2}\right\|_{\mathbb{W}} \leq c|\mu|_{L^{\infty}\left(\Gamma_{C}\right)}\|g\|_{\mathbb{W}}
$$

and, using (5.1), we deduce that

$$
\left\|\Lambda g^{1}-\Lambda g^{2}\right\|_{\mathbb{W}} \leq c|\mu|_{L^{\infty}\left(\Gamma_{C}\right)}\left\|g^{1}-g^{2}\right\|_{\mathbb{W}} .
$$

We choose $\mu_{0}=1 / c$, and Proposition 5.1 is now a consequence of (5.13) and the Banach fixed point theorem.

Proof of Theorem 2.2. Using (5.1) and Proposition 5.1, it is straightforward to show that the solution $\left\{\mathbf{u}_{g^{*}}, \sigma_{g^{*}}, \xi_{g^{*}}\right\}$ of Problem $P_{g^{*}}$ is a solution of Problem $P$ when $|\mu|_{L^{\infty}\left(\Gamma_{C}\right)}$ is sufficiently small. The uniqueness part in Theorem 2.2 can be deduced from the uniqueness of the solution of Problem $P_{g^{*}}$ and the uniqueness of the fixed point operator $\Lambda$. 


\section{REFERENCES}

[1] R. S. Adams, Sobolev Spaces, Pure and Applied Mathematics, vol. 65, Academic Press, New York and London, 1975

[2] L.-E. Andersson, A quasistatic frictional problem with normal compliance, Nonlinear Anal. 16(4), 347-369 (1991)

[3] K. T. Andrews, P. Shi, M. Shillor, and S. Wright, Thermoelastic contact with Barber's heat exchange condition, Appl. Math. Opt. 28(1), 1148 (1993)

[4] K. T. Andrews, K. L. Kuttler, and M. Shillor, On the dynamic behaviour of a thermoviscoelastic body in frictional contact with a rigid obstacle, European J. Appl. Math. 8, 417-436 (1997)

[5] K. T. Andrews, A. Klarbring, M. Shillor, and S. Wright, On the the dynamic behavior of a thermoviscoelastic contact problem with friction and wear, Internat. J. Engrg. Sci. 35(14), 12911309 (1997)

[6] J.-P. Aubin, Approximation of Elliptic Boundary Value Problems, John Wiley and Sons, New York, 1972

[7] M. Cocu, E. Pratt, and M. Raous, Formulation and approximation of quasistatic frictional contact, Internat. J. Engrg. Sci. 34(7), $783-798$ (1996)

[8] G. Duvaut, Loi de frottement non locale, J. Méc. Thé. Appl., Special issue, 73-78 (1982)

[9] G. Duvaut and J. L. Lions, Les Inéquations en Mécanique et en. Physique, Dunod, 1972

[10] I. Figueiro and L. Trabucho, A class of contact and friction dynamic problems in thermoelasticity and in thermoviscoelasticity, Internat. J. Engrg. Sci. 33(1), 45-66 (1995)

[11] R. J. Gu, K. L. Kuttler, and M. Shillor, Frictional wear of a thermoelastic beam, J. Math. Anal. Appl. 242, 212-236 (2000)

[12] R. J. Gu and M. Shillor, Thermal and wear finite elements analysis of an elastic beam in sliding contact, Internat. J. Solids Structures, to appear

[13] L. Johansson and A. Klarbring, Thermoelastic frictional contact problems: Modelling, finite element approximation and numerical realization, Comput. Methods Appl. Mech. Engrg. 105, 181-210 (1993)

[14] K. L. Kuttler, Time-dependent implicit evolution equations, Nonlinear Anal. 10(5), 447-463 (1986)

[15] K. L. Kuttler, Y. Renard, and M. Shillor, Models and simulations of dynamic frictional contact of a beam. Computational modeling of contact and friction, Comput. Methods Appl. Mech. Engrg. 177, 259-272 (1999)

[16] K. L. Kuttler and M. Shillor, Set-valued pseudomonotone maps and degenerate evolution inequalities, Commun. Contemp. Math. 1, 87--123 (1999)

[17] N. Kikuchi and T. J. Oden, Contact Problems in Elasticity, SIAM, 1988

[18] A. Klarbring, A. Mikelic, and M. Shillor, A global existence result for the quasistatic frictional contact problem with normal compliance, pp. 85-111 in Unilateral Problems in Structural Mechanics $I V$, eds. G. DelPiero and F. Maceri, Birkhäuser, Boston, 1991

[19] J. L. Lions, Quelques Méthodes de Résolution des Problèmes aux Limites non Linéaires, Dunod, 1969

[20] M. Rochdi and M. Shillor, A dynamic thermoviscoelastic frictional contact problem with damped response, preprint, 1998

[21] M. Rochdi, M. Shillor, and M. Sofonea, Quasistatic viscoelastic contact with normal compliance and friction, J. Elasticity 51, 105-126 (1998)

[22] M. Rochdi, M. Shillor, and M. Sofonea, A quasistatic contact problem with directional friction and damped response, Applicable Analysis 68(3-4), 409-422 (1998)

[23] M. Shillor, editor, Contact Mechanics, a special issue of Computer and Math. Modelling 28, 4-8 (1998)

[24] M. Shillor and M. Sofonea, A quasistatic viscoelastic contact problem with friction, Internat. J. Engrg. Sci., to appear

[25] N. Strömberg, L. Johansson, and A. Klarbring, Derivation and analysis of a generalized standard model for contact friction and wear, Internat. J. Solids Structures 33(13), 1817-1836 (1996) 